\title{
Activated protein $C$ modulates the proinflammatory activity of dendritic cells
}

This article was published in the following Dove Press journal:

Journal of Asthma and Allergy

7 May 2015

Number of times this article has been viewed

\author{
Takahiro Matsumoto ${ }^{1,2, *}$ \\ Yuki Matsushima ${ }^{1, *}$ \\ Masaaki Toda' \\ Ziaurahman Roeen' \\ Corina N D'Alessandro- \\ Gabazza ${ }^{1,5}$ \\ Josephine A Hinneh' \\ Etsuko Harada ${ }^{1,3}$ \\ Taro Yasuma ${ }^{4}$ \\ Yutaka Yano ${ }^{4}$ \\ Masahito Urawa ${ }^{1,5}$ \\ Tetsu Kobayashi ${ }^{5}$ \\ Osamu Taguchi ${ }^{5}$ \\ Esteban C Gabazza' \\ 'Department of Immunology, Mie \\ University Graduate School of \\ Medicine, Tsu, Mie Prefecture, \\ ${ }^{2}$ BONAC Corporation, BIO Factory \\ 4F, Fukuoka, ${ }^{3}$ wade Research \\ Institute of Mycology, ${ }^{4}$ Department \\ of Endocrinology, Diabetes and \\ Metabolism, ${ }^{5}$ Department of \\ Pulmonary and Critical Care \\ Medicine, Mie University Graduate \\ School of Medicine, Tsu, Mie \\ Prefecture, Japan
}

*These authors contributed equally to this work

Correspondence: Esteban C Gabazza Department of Immunology, Mie University Graduate School of Medicine, Edobashi 2-174, 5I4-8507, Tsu, Mie Prefecture, Japan

$\mathrm{Tel}+8|5923| 5017$

Fax +8I 59 23I 5225

Email gabazza@clin.medic.mie-u.ac.jp
Background: Previous studies have demonstrated the beneficial activity of activated protein $\mathrm{C}$ in allergic diseases including bronchial asthma and rhinitis. However, the exact mechanism of action of activated protein $\mathrm{C}$ in allergies is unclear. In this study, we hypothesized that pharmacological doses of activated protein $\mathrm{C}$ can modulate allergic inflammation by inhibiting dendritic cells.

Materials and methods: Dendritic cells were prepared using murine bone marrow progenitor cells and human peripheral monocytes. Bronchial asthma was induced in mice that received intratracheal instillation of ovalbumin-pulsed dendritic cells.

Results: Activated protein C significantly increased the differentiation of tolerogenic plasmacytoid dendritic cells and the secretion of type I interferons, but it significantly reduced lipopolysaccharide-mediated maturation and the secretion of inflammatory cytokines in myeloid dendritic cells. Activated protein $\mathrm{C}$ also inhibited maturation and the secretion of inflammatory cytokines in monocyte-derived dendritic cells. Activated protein C-treated dendritic cells were less effective when differentiating naïve CD4 T-cells from Th1 or Th2 cells, and the cellular effect of activated protein $\mathrm{C}$ was mediated by its receptors. Mice that received adoptive transfer of activated protein C-treated ovalbumin-pulsed dendritic cells had significantly less airway hyperresponsiveness, significantly decreased lung concentrations of Th1 and Th2 cytokines, and less plasma concentration of immunoglobulin $\mathrm{E}$ when compared to control mice.

Conclusion: These results suggest that dendritic cells mediate the immunosuppressive effect of activated protein $\mathrm{C}$ during allergic inflammation.

Keywords: allergy, dendritic cells, coagulation, protein $\mathrm{C}$ pathway

\section{Introduction}

Dendritic cells (DCs) are the strongest professional antigen-presenting cells that differentiate from bone marrow and migrate to several organs, where they interrogate foreign antigens. ${ }^{1}$ After antigen phagocytosis, DCs are activated and move to the draining lymph nodes, where they present the antigen to cognate T-cells to stimulate immune responses. ${ }^{1}$ The three main DC subsets are the plasmacytoid DCs (CD11b ${ }^{-}$, CD45R ${ }^{+}$) and the type 1 and type 2 myeloid or conventional DCs (CD11b $\left.\mathrm{b}^{+}, \mathrm{CD} 45 \mathrm{R}^{-}\right)$; plasmacytoid DCs are known to interact with T-cells to induce tolerance, whereas conventional DCs are efficient in priming naïve T-cells to induce acquired immune reactions. ${ }^{1,2}$ However, both plasmacytoid DC and conventional DC subsets can be tolerogenic or immunogenic depending on the microenvironmental conditions. ${ }^{1-3}$

Activated protein C (APC) is a natural anticoagulant that plays a fundamental role as an inhibitor of the coagulation system by inactivating the procoagulant factors Va and 
VIIIa. ${ }^{4}$ Previous in vitro and in vivo studies have shown that APC plays an important role in the modulation of inflammatory and immune responses, wound healing, and apoptosis. ${ }^{5}$ This protective activity of APC has been shown to depend on different mechanisms including the reduction of the expression of inflammatory cytokines, adhesion molecules, and growth factors from different cells; protection of the endothelial barrier function; blockade of the activation and extravascular migration of leukocytes at sites of tissue injury; and the inhibition of cell apoptosis. ${ }^{6}$ These cellular effects are mediated by the APC receptors, endothelial protein $C$ receptor (EPCR), protease-activated receptors (PARs), and/or CD11b, though the exact intracellular signaling pathways involved in this receptor-mediated action of APC remain unclear. ${ }^{6}$

In the present study, we hypothesized that pharmacological doses of APC can modulate inflammatory and immune responses by inhibiting the differentiation and maturation of DCs. To demonstrate this hypothesis, we evaluated the effect of APC using murine and human DCs.

\section{Materials and methods}

\section{Reagents}

L-glutamine, penicillin/streptomycin, and TRIzol ${ }^{\circledR}$ reagent were purchased from Thermo Fisher Scientific (Waltham, MA, USA); Roswell Park Memorial Institute (RPMI)-1640, and bovine serum albumin were from Sigma-Aldrich Co (St Louis, MO, USA); and fetal bovine serum (FBS) was from BioWhittaker, Inc. (Walkersville, MD, USA). Human APC was a kind gift from Kaketsuken (Kumamoto, Japan). Purification (purity $>95 \%$ ) and the assays for the activity of APC and for the absence of thrombin activity was performed as previously described. ${ }^{7}$

\section{Cell culture}

For the generation of bone marrow-derived DCs, murine bone marrow cells from C57BL/6 wild-type mice were cultivated for 7 days at $37^{\circ} \mathrm{C}$ in a humidified atmosphere containing $5 \% \mathrm{CO}_{2}$ in RPMI-1640 supplemented with 10\% heat-inactivated FBS, $2 \mathrm{mM}$ of L-glutamine, $100 \mathrm{U} / \mathrm{mL}$ of penicillin, $100 \mu \mathrm{g} / \mathrm{mL}$ of streptomycin, $50 \mu \mathrm{M}$ of $\beta$-mercaptoethanol (Sigma-Aldrich Co), and 200 ng/mL of Flt3L (PeproTech, Inc., Rocky Hill, NJ, USA). ${ }^{8}$ For the generation of human monocyte-derived DCs, monocytes prepared from human peripheral blood were cultured for 6 days in the presence of $50 \mathrm{ng} / \mathrm{mL}$ of human recombinant granulocyte macrophage colony-stimulating factor (GM-CSF) (PeproTech, Inc.) and $50 \mathrm{ng} / \mathrm{mL}$ of human recombinant interleukin (IL)-4 (PeproTech, Inc.). ${ }^{9}$ APC was added to a final concentration of $20 \mu \mathrm{g} / \mathrm{mL}$ or $40 \mu \mathrm{g} / \mathrm{mL}$ on day 4 (to see the effect on DC differentiation) or on day 6 (to see the effect on DC maturation) of bone marrow-derived DC culture. The maturation of DCs was induced by adding $100 \mu \mathrm{g} / \mathrm{mL}$ of ovalbumin (OVA) or $100 \mathrm{ng} / \mathrm{mL}$ of Escherichia coli lipopolysaccharide (LPS) (Sigma-Aldrich Co) on day 6 , and by continuing the culture for 24 hours. Neutralizing antibodies were added 30 minutes before the APC treatment at a final concentration of $5 \mu \mathrm{g} / \mathrm{mL}$. The antibodies used to block the APC receptors were as follows: antimouse EPCR (rat immunoglobulin [Ig]G2a, clone: RCR16; BioLegend, Inc., San Diego, CA, USA); antihuman EPCR (rat IgG1, clone: RCR252; BD Biosciences, San Jose, CA, USA); antimouse PAR-1 (rabbit IgG, polyclonal; Santa Cruz Biotechnology, Inc., Dallas, TX, USA); antihuman PAR-1 (mouse IgG1, clone: ATAP2; Santa Cruz Biotechnology, Inc.); and antimouse and human CD11b (rat IgG2b, M1/70). The Committee for Animal Investigation of Mie University (Tsu, Mie Prefecture, Japan) approved the experimental protocol (approval number: 24-50). Written informed consent was obtained from all healthy volunteers and the protocol was approved by the Ethics Board of Mie University for Clinical Investigation (approval 2707).

\section{Preparation and purification of antigen-specific CD4 T-cells}

Naïve $\mathrm{CD} 4^{+} \mathrm{T}$-cells recognizing a peptide of chicken OVA in the context of $\mathrm{I}-\mathrm{A}^{\mathrm{b}}$ were isolated from the spleens of OT-II T-cell receptor (TCR) transgenic mice (C57BL/6 background) following instructions in the naïve CD4 T-cell isolation kit II (Miltenyi Biotec GmbH, Bergisch Gladbach, Germany).

\section{Antigen-presentation assay}

Bone marrow-derived DCs were prepared from C57BL/6 mice and cultured in the presence of recombinant Flt3L, and then varying doses of APC were added to the culture on day 6 and stimulated with $100 \mu \mathrm{g} / \mathrm{mL}$ of OVA for 24 hours. ${ }^{10}$ CD11 $\mathrm{c}^{+}$DCs were purified using mouse CD11c microbeads (Miltenyi Biotec $\mathrm{GmbH}$ ). Naïve CD4 T-cells were prepared from OT-II mice and $1 \times 10^{5}$ cells were stimulated with $1 \times 10^{4}$ CD11 $\mathrm{c}^{+}$DCs either in the Th1-skewing condition (in the presence of $50 \mathrm{ng} / \mathrm{mL}$ of IL-2 and $5 \mu \mathrm{g} / \mathrm{mL}$ of anti-IL4) or the Th2-skewing condition (in the presence of $50 \mathrm{ng} / \mathrm{mL}$ of IL-4 and $5 \mu \mathrm{g} / \mathrm{mL}$ of anti-IL-12).

\section{Flow cytometry}

If not noted otherwise, antibodies were acquired from BD Biosciences. Bone marrow-derived DCs were incubated 
with the anti-CD16/CD32 FcR block (rat IgG2b, clone: 2.4G2) and stained with antimouse CD11c (Armenian hamster IgG1, clone: HL3), antimouse CD11b (rat IgG2b, clone: M1/70), antimajor histocompatibility complex (MHC) II (I-A/I-E, rat IgG2b, clone: M5.114), antimouse CD86 (rat IgG2a, clone: GL-1), antimouse CD80 (rat IgG2a, clone: 1G10, Southern Biotechnology Associates, Inc., Birmingham, AL, USA), antimouse CD45R (rat IgG2a, clone: RA3-6B2), or their isotype controls. Human monocyte-derived DCs were incubated with human IgG to block FcR and they were stained with antimouse CD11c (mouse IgG1, clone: B-ly6), anti-HLA-DR (mouse IgG2a, clone: L243), antihuman CD86 (mouse IgG1, clone: 2331), and antihuman CD80 (mouse IgG1, clone: L307.4), or their isotype controls. After 20 minutes of incubation at $4^{\circ} \mathrm{C}$, the cells were washed twice in fluorescence activated cell sorting (FACS) buffer ( $1 \%$ fetal calf serum [FCS] and $0.1 \%$ $\mathrm{NaN}_{3}$ in phosphate buffered saline [PBS]) and analyzed by FACScan flow cytometer (BD, Franklin Lakes, NJ, USA) using the Cell-Quest Pro software (BD).

For intracellular cytokine staining, cells were stimulated with phorbol 12-myristate 13-acetate/ionomycin in the presence of brefeldin A for the final 3 hours. Cells were incubated in the presence of $25 \mu \mathrm{g}$ of anti-CD16/CD32 for 10 minutes at $4^{\circ} \mathrm{C}$, washed once in FACS buffer ( $1 \% \mathrm{FCS}$ and $0.1 \% \mathrm{NaN}_{3}$ in PBS), and fixed in $4 \%$ paraformaldehyde in PBS. Subsequently, cells were incubated in permeabilization buffer $(0.5 \%$ saponin, $1 \%$ FCS, and $2 \mathrm{mM} 4$-[2-hydroxyethyl]-1-piperazineethanesulfonic acid [HEPES] in PBS) for 20 minutes and stained for intracellular cytokines with the monoclonal antibodies, antimouse interferon (IFN) $\gamma$ (rat IgG1, clone: XMG1.2), antimouse IL-4 (rat IgG1, clone: 11B11), and antimouse IL-10 (rat IgG2b, clone: JES5-16E3), or their isotype controls.

\section{Semiquantitative reverse transcription polymerase chain reaction (RT-PCR) analysis}

Total RNA was extracted from the cells by the guanidine isothiocyanate procedure using the TRIzol ${ }^{\circledR}$ Reagent (Thermo Fisher Scientific), reverse-transcribed using oligo-dT primers, and then the DNA was amplified by polymerase chain reaction (PCR). The sequences of the primers are described in Table 1. PCR was carried for 25 35 cycles depending on the gene, denaturation was performed at $94^{\circ} \mathrm{C}$ for 30 seconds, annealing at $59^{\circ} \mathrm{C} \sim 65^{\circ} \mathrm{C}$ for 30 seconds, and elongation at $72^{\circ} \mathrm{C}$ for 1 minute; at the end of these cycles, a further extension was carried out at $72^{\circ} \mathrm{C}$ for 5 minutes. The PCR products were separated on a $2 \%$ agarose gel containing $0.01 \%$ ethidium bromide. The concentration of RNA and its purity were determined by ultraviolet (UV) absorption at 260:280 using an Ultrospec 1100 pro UV/Vis spectrophotometer (Amersham Biosciences Corp, Picastaway, NJ, USA). The messenger (m)RNA amount was normalized for the glyceraldehyde 3-phosphate dehydrogenase mRNA and expressed as the percent of control.

\section{Animals}

The Mie University Committee on animal research approved the protocol where experimental animals were used. All experiments were carried out following the guidelines for animal experiments of the National Institute of Health. Wild-type female C57BL/6 mice were purchased from Nihon SLC (Hamamatsu, Japan). OT-II TCR transgenic mice were from K Takahashi, Yokohama City University Graduate School of Medicine (Yokohama, Japan). Mice were bred at Mie University Animal Experimental Center in a

Table I Sequence of primers used in the study

\begin{tabular}{|c|c|c|}
\hline Gene & Sense & Antisense \\
\hline mGAPDH & 5'-CCTTATTGACCTCAACTACATGGT-3' & 5'-GAGGGGCCATCCACAGTCTTCTG-3' \\
\hline$m I F N-\gamma$ & 5'-GCTCTGACACAATGAACGCT-3' & 5'-AAAGAGATAATCTGGCTCTGC-3' \\
\hline$m I F N-\alpha$ & 5'-ATGGCTAGCTCTGTGCTTTCCT-3 & 5'-AGGGCTCTCCAGATTCTGCTCTG-3 \\
\hline mIFN- $\beta$ & 5'-CATCAACTATAAGCAGCTCCA-3 & 5'-TTCAAGTGGAGAGCAGTTGAG-3 \\
\hline mIL-4 & 5'-ATGGGTCTCAACCCCCAGCTAGT-3' & 5'-GCTCTTTAGGCTTTCCAGGAAGTC-3' \\
\hline mIL-10 & 5'-TCAAACAAAGGACCAGCTGGACAACATACTG-3' & 5'-CTGTCTAGGTCCTGGAGTCCAGCAGACTCA-3' \\
\hline mPAR-I & 5'-GGTCTGCTACACGTCCATCAT-3' & 5'-AGCACAAGATGCTGTAGAGGT-3' \\
\hline mPAR-2 & 5'-CAAGGTGCTCATTGGCTTTT-3' & 5'-CAGAGGGCGACAAGGTAGAG-3' \\
\hline mPAR-3 & 5'-ATCTCAATGGCAACAACTGG-3' & 5'-AGGAACCCAAAGAATGCTAA-3' \\
\hline mPAR-4 & 5'-ACGCTCACTACTGGACTCT-3' & 5'-AGGGCTCGGGTTTTGAATAGT-3' \\
\hline$m E P C R$ & 5'-GAAAGGGAGCCAAACAGGTC-3' & 5'-ACCAGCCCCCACCAGTCCA-3' \\
\hline hGAPDH & 5'-CCACCCATGGCAAATTCCATGGCA-3' & 5'-TCTAGACGGCAGGTCAGGTCCACC-3' \\
\hline hTNF- $\alpha$ & 5'-GAAAGCATGATCCGGGACGT-3' & 5'-CTTGGTCTGGTAGGAGACGG-3' \\
\hline
\end{tabular}


specific-pathogen-free environment, fed a normal laboratory diet, and subjected to a 12-hour light:dark cycle.

\section{Adoptive transfer of sensitized DCs and disease model preparation}

Murine bone marrow-derived DCs were prepared as described earlier, and then nonadherent cells were collected and cultured in the presence of saline alone, OVA $(250 \mu \mathrm{g} / \mathrm{mL})$ alone, or OVA plus APC $(60 \mu \mathrm{g} / \mathrm{mL})$ for 24 hours. The DCs were then administered to female C57BL/6 mice by intratracheal instillation and, a week later, each group of mice was challenged with aerosolized 4\% OVA for 3 consecutive days in an exposure chamber (MIPS, KK, Osaka, Japan). Airway hyperresponsiveness was measured using a whole-body plethysmograph system (Buxco Electronics, Inc., Sharon, CT, USA) before sacrifice by pentobarbital overdose.

\section{Bronchoalveolar lavage fluid (BALF) sampling and biochemical analysis}

BALF was sampled as previously described. ${ }^{7,11}$ The cytokine concentrations in cell supernatants and BALF, as well as the plasma concentration of $\operatorname{IgE}$ were measured by commercially available sandwich enzyme-linked immuno- sorbent assay kits (BD Biosciences) following the manufacturer's instructions.

\section{Statistics}

The statistical difference between several groups was evaluated by the Kruskal-Wallis one-way analysis of variance with post hoc analysis using Dunn's test. The nonparametric Mann-Whitney $U$ test was used to compare the results between two groups. Statistical significance was considered as a $P$-value $<0.05$.

\section{Results \\ APC promotes expansion of plasmacytoid DCs}

We first tested the hypothesis that APC affects the differentiation of DCs from bone marrow cells. Bone marrow-derived DCs were prepared from C57BL/6 mice and cultured in the presence of recombinant Flt3L, and then $20 \mu \mathrm{g} / \mathrm{mL}$ of APC was added to the culture on day 4 and further cultured for 3 days. The percentage of plasmacytoid DCs was significantly increased, and the percentage of conventional DCs and the ratio of conventional DCs to plasmacytoid DCs were significantly decreased in the presence of APC compared to
A

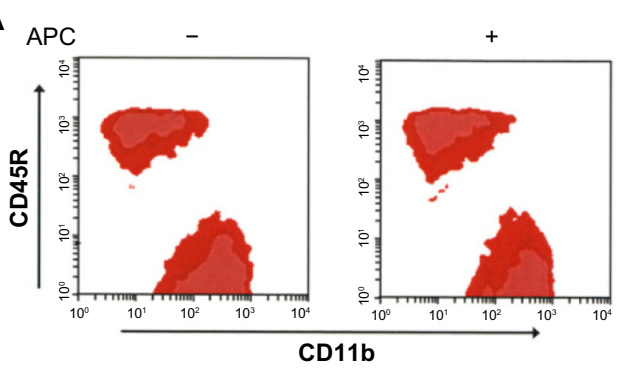

CD11b
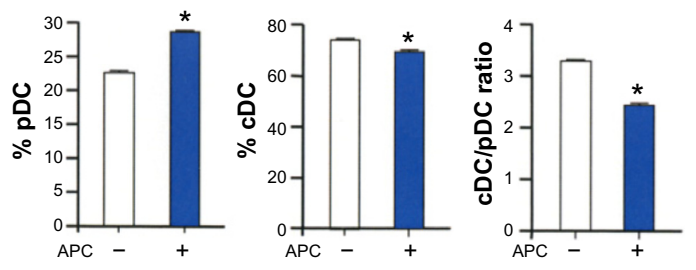

B

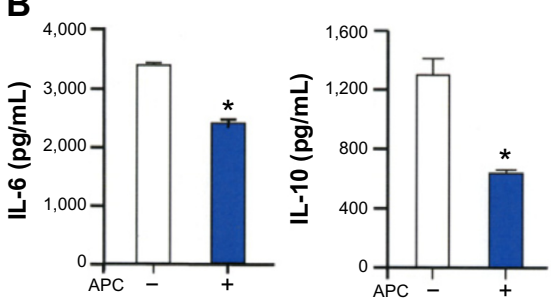

C

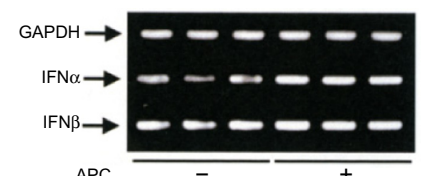

APC
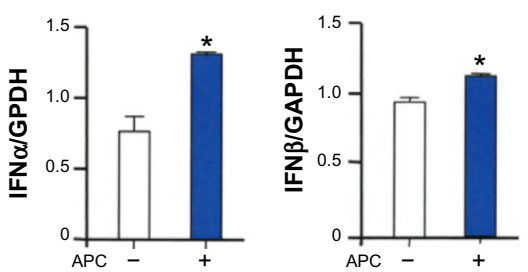

Figure I APC promotes the expansion of plasmacytoid DCs.

Notes: Bone marrow cells were prepared from C57BL/6 mice and cultured in the presence of Flt $3 \mathrm{~L}$, and then $20 \mu \mathrm{g} / \mathrm{mL}$ of APC was added to the culture on day 4 and

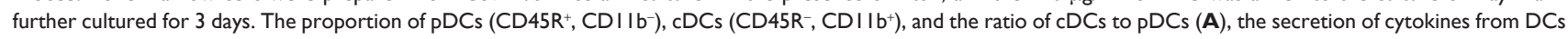
(B), and the RT-PCR analysis of IFN $\alpha$ and IFN $\beta$ (C) are described. The data are expressed as the mean \pm standard error of the mean. The figure shows the representative result from one of three independent experiments. $* P<0.05$ compared to the untreated group.

Abbreviations: APC, activated protein C; IL, interleukin; DCs, dendritic cells; cDC, conventional dendritic cells; pDCs, plasmacytoid dendritic cells; GAPDH, glyceraldehyde 3-phosphate dehydrogenase; IFN, interferon; RT-PCR, reverse transcription polymerase chain reaction. 
control cells (Figure 1A). Also, the expression of IL-6 and IL-10 was significantly decreased, while that of IFN $\alpha$ and IFN $\beta$ was significantly increased in DCs treated with APC compared to control cells (Figure 1B and C).

\section{APC inhibits maturation of bone marrow-derived DCs}

We then investigated the effect of APC on the maturation of DCs. Bone marrow-derived DCs were prepared from $\mathrm{C} 57 \mathrm{BL} / 6$ mice and cultured in the presence of recombinant human Flt3L, and then $40 \mu \mathrm{g} / \mathrm{mL}$ of APC or heat-denatured APC was added to the culture on day 6 and stimulated with LPS for 24 hours. The maturation markers MHC class II, CD86, and CD80 were significantly decreased in cells cultured in the presence of APC, but not by heat-denatured APC, compared to control cells (Figure 2A and B). A lower concentration of APC $(20 \mu \mathrm{g} / \mathrm{mL})$ also tended to decrease the maturation of DCs, but not at significant level (Figure 2C). The protein secretions of IL-12p70 and IL- 6 were also significantly decreased in the presence of APC when compared to controls (Figure 2C).

\section{Inhibition of maturation of human monocyte-derived DCs by APC}

We assessed whether APC can also affect human DCs. Monocytes from human peripheral blood were treated with recombinant GM-CSF and IL-4 before adding APC on day 6, and they were stimulated with LPS for 24 hours. The suppressive activity of APC on the DC expression of HLA-DR, CD86, and CD80 was weak but statistically significant compared to untreated cells (Figure 3A). APC significantly reduced the expression of tumor necrosis factor (TNF)- $\alpha$ from human monocyte-derived DCs compared to controls (Figure 3B).

\section{Bone marrow-derived DCs treated with APC are less effective to prime naïve T-cells}

The ability of APC-DCs to prime naïve T-cells was assessed. Bone marrow-derived DCs were prepared from C57BL/6 mice, cultured in the presence of recombinant human Flt3L, and then varying doses of APC were added to the culture on day 6 and stimulated with OVA for 24 hours; naïve CD4 T-cells from OT-II transgenic mice were then cocultured with $\mathrm{CD} 11 \mathrm{c}^{+} \mathrm{DCs}$ either

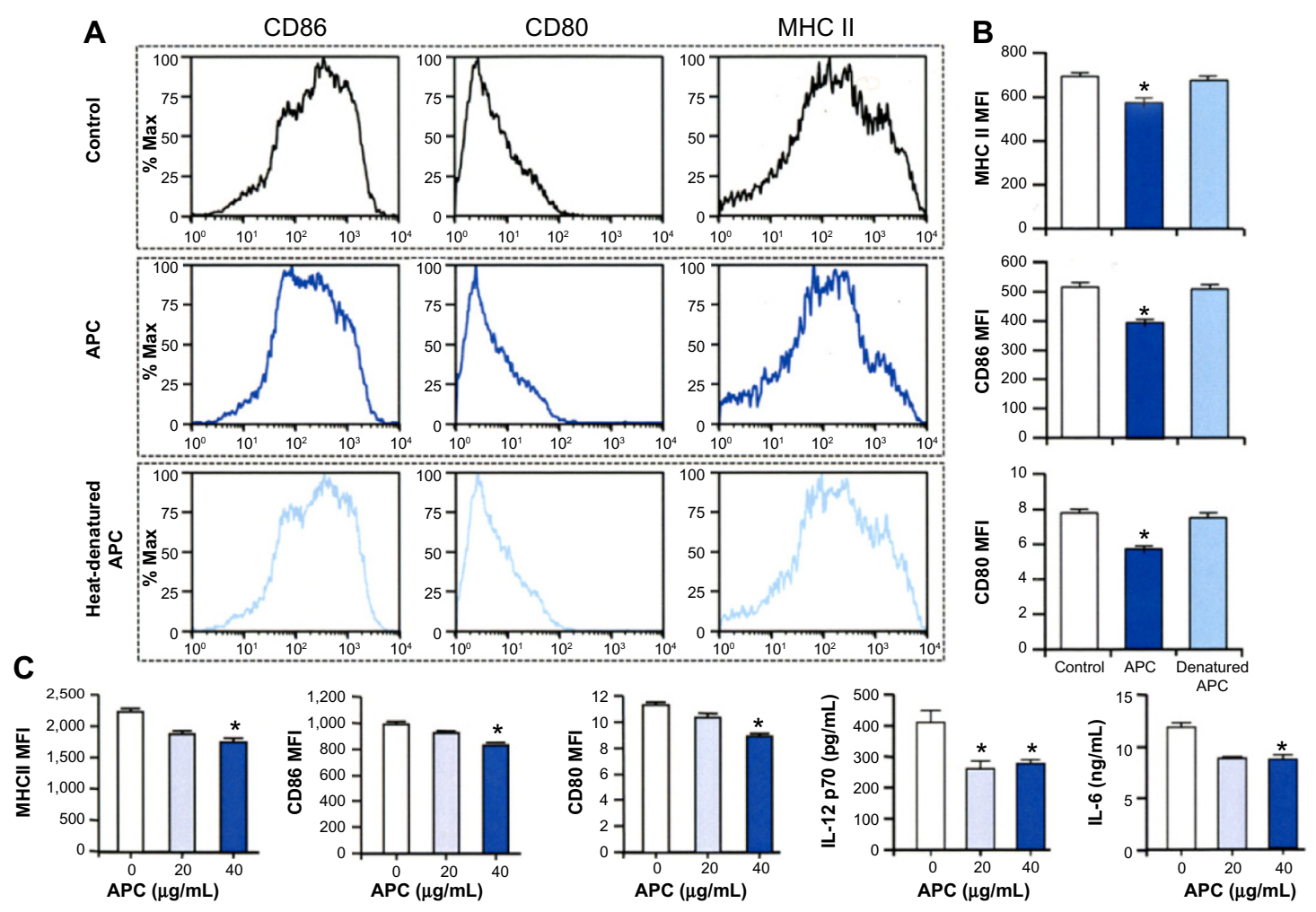

Figure 2 APC inhibits maturation of bone marrow-derived DCs.

Notes: Bone marrow cells were prepared from C57BL/6 mice, cultured in the presence of Flt3L, and then APC was added to the culture on day 6 and stimulated with LPS for 24 hours. The expression of MHC class II, CD86, and CD80 on CDI Ic ${ }^{+}$DCs in the presence of $40 \mu \mathrm{g} / \mathrm{mL}$ of APC (A) and the mean fluorescent intensity were measured (B). The effects of different doses of APC on maturation markers and secretion of cytokines from DCs (C) are also shown. Data are expressed as the mean \pm standard error of the mean $(n=3)$. The figure shows the representative result from one of three independent experiments. $* P<0.05$ compared to the untreated group.

Abbreviations: APC, activated protein C; MHC, major histocompatibility complex; MFI, mean fluorescence intensity; IL, interleukin; DCs, dendritic cells; LPS, lipopolysaccharide; $\mathrm{n}$, number. 

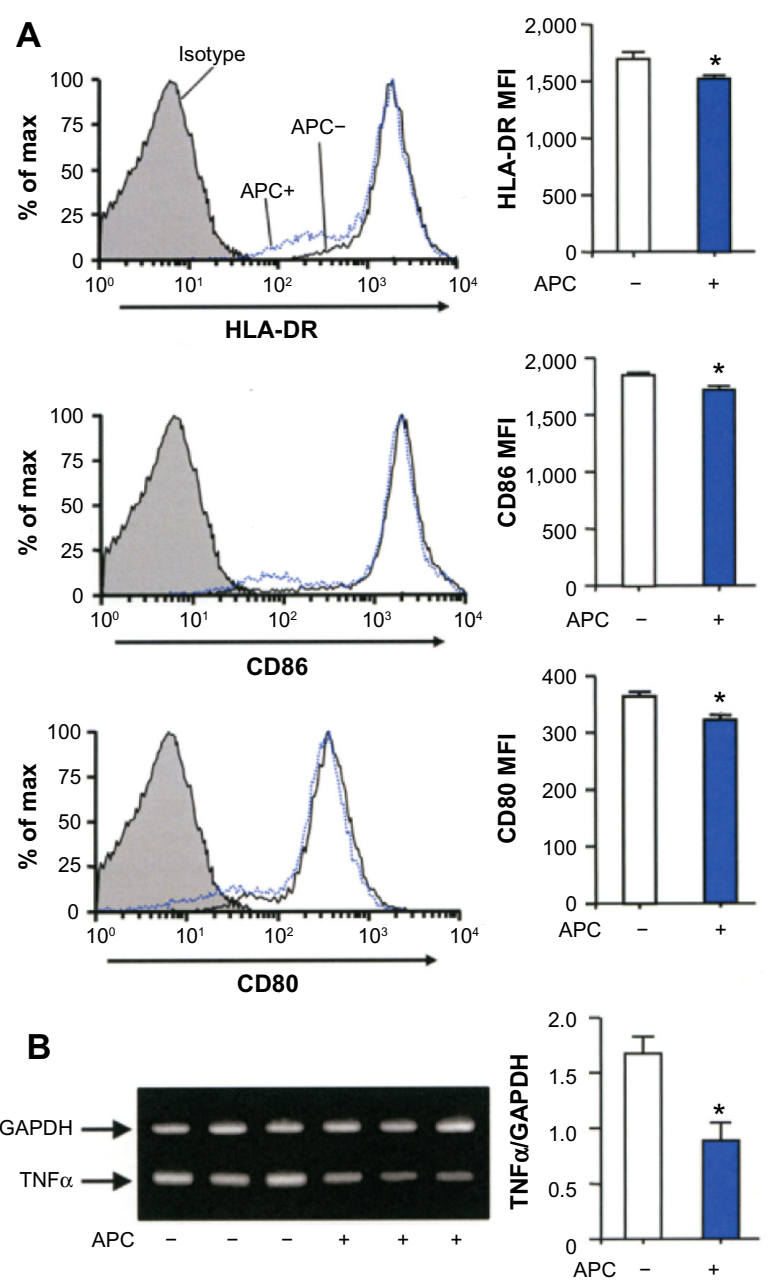

Figure 3 APC inhibits the maturation of human monocyte-derived DCs. Notes: Monocytes were prepared from human peripheral blood and cultured in the presence of recombinant GM-CSF and IL-4, and then APC $(40 \mu \mathrm{g} / \mathrm{mL})$ was added to the culture on day 6 and stimulated with LPS for 24 hours. The expression of HLADR, CD86, and CD80 on CDIIc $c^{+}$DCs in the presence or absence of APC (A), as well as the secretion of TNF $\alpha$ from DCs $(\mathbf{B})$ are shown. Data are expressed as the mean \pm standard error of the mean. The figure shows the representative result from one of three independent experiments. $* P<0.05$ compared to the untreated group. Abbreviations: Max, maximum; APC, activated protein C; MFI, mean fluorescence intensity; GAPDH, glyceraldehyde 3-phosphate dehydrogenase; TNF $\alpha$, tumor necrosis factor alpha; DCs, dendritic cells; GM-CSF, granulocyte macrophage colony-stimulating factor; IL, interleukin; LPS, lipopolysaccharide.

in the Th1-skewing condition or the Th2-skewing condition. APC significantly inhibited the number of IFN $\gamma^{+}, \mathrm{IL}_{-} 4^{+}$, and IL-10 ${ }^{+}$T-cells compared to controls (Figure 4A and B).

\section{APC receptors mediate APC-mediated regulation of DCs}

Mature bone marrow-derived DCs express EPCR, as well as the PAR-1, PAR-2, PAR-3, and PAR-4 by RT-PCR, and the expressions of EPCR, PAR-1, and CD11b were confirmed by flow cytometry (Figure 5A). Pretreatment of OVA-pulsed CD11 ${ }^{+}$DCs with anti-EPCR, anti-PAR-1, or anti-CD11b antibody before stimulation with APC and coculture with CD4
A Th1-skewing condition

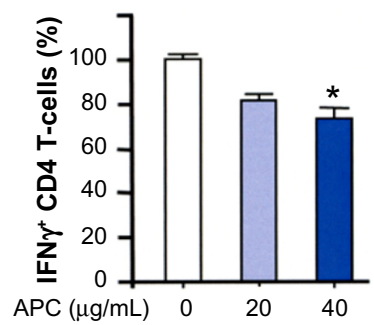

B Th2-skewing condition

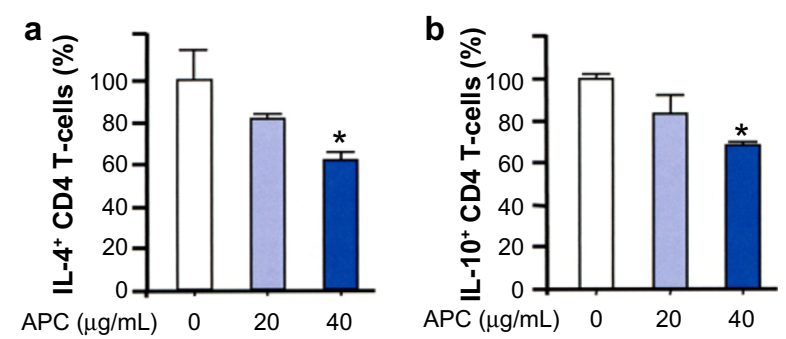

Figure 4 APC-treated bone marrow-derived DCs are less effective to prime naïve CD4 T-cells.

Notes: Bone marrow cells were prepared and cultured in the presence of recombinant human Flt3L; then, doses of APC were added. Naïve CD4 T-cells were prepared and then stimulated with CDII c $\mathrm{c}^{+} \mathrm{DCs}$ either in the ThI-skewing condition (A) or the Th2-skewing condition (B). The relative proportion of IFN $\gamma^{+}$CD4 T-cells in the ThI-skewing condition, and IL- $4^{+}$and IL- $10^{+}$CD4 T-cells in Th2-skewing condition are shown. Data are expressed as the mean \pm standard error of the mean. The figure shows the representative result from one of the three independent experiments. $* P<0.05$ compared to the untreated group.

Abbreviations: IFN, interferon; APC, activated protein C; IL, interleukin; DCs, dendritic cells.

T-cells significantly inhibited the suppression of the IFN $\gamma^{+}$ CD4 T-cell generation by APC. The inhibition of protein and RNA expression of IFN $\gamma$ (Figure 5B) and the RNA expression of IL-4 and IL-10 from T-cells (Figure 5C) by APC were also blocked by treatment with anti-APC receptor antibodies.

\section{APC inhibits the proinflammatory activity of OVA-pulsed bone marrow-derived DCs in vivo}

The effect of APC on allergic inflammation in vivo was evaluated. Pretreatment of OVA-pulsed bone marrowderived DCs with APC before intratracheal instillation into mice was associated with significant inhibition of airway hyperresponsiveness and a significantly lower concentration of IFN $\gamma$ and IL-5 in BALF as compared to controls (Figure 6). The plasma concentration of IgE was decreased in mice that were pretreated with APC-pulsed DCs when compared to control mice, but not at a significant level (Figure 6).

\section{Discussion}

This study demonstrated that APC can regulate the inflammatory and immune response induced by DCs. 
A

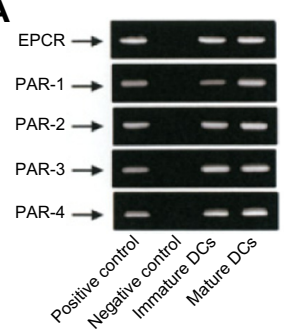

CD11b

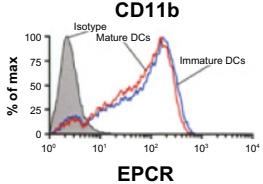

EPCR

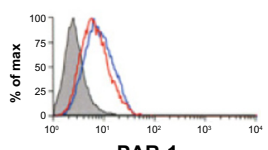

PAR-1

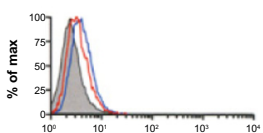

B
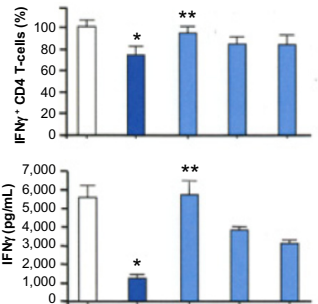

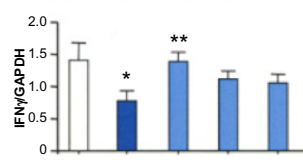

$A P C$
Anti-EPCR
Ant

Anti-EPCR
Anti-PAR-1
Ant-CDis

Anti-PAR-1
Anti-CD11b

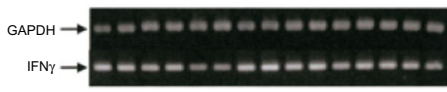

APC

APC
Anti-EPCR
Anti-PAR-1
-1

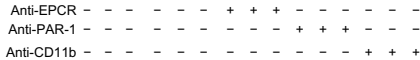

Figure 5 Mediation of APC receptors in the bone marrow-derived DC-mediated priming of T-cells.

Notes: Bone marrow-derived DCs were prepared and cultured in the presence of recombinant Flt3L for 6 days and stimulated with LPS for 24 hours. Total RNA was prepared from immature (LPS-untreated) and mature (LPS-treated) DCs and analyzed for the expression of EPCR, PAR-I, PAR-2, PAR-3, and PAR-4 by RT-PCR (A). Expressions of EPCR, PAR-I, and CDI Ib on the cell surface of DCs were analyzed by flow cytometry (A). For T-cell priming assays, naïve CD4 T-cells were prepared and then cultured with CDI I c ${ }^{+}$DCs. The relative proportion of IFN $\gamma^{+}$CD4 T-cells and IFN $\gamma$ secretion (B), as well as RNA expression of IFN $\gamma$, IL-4, and IL-I0 (C) are shown. Data are expressed as the mean \pm standard error of the mean $(n=3)$. The figure shows the representative result from one of two independent experiments. $* P<0.05$ compared to the untreated group. ${ }^{*} * P<0.01$ compared to the group treated with APC alone.

Abbreviations: EPCR, endothelial protein C receptor; PAR, protease-activated receptor; DCs, dendritic cells; Max, maximum; IFN, interferon; GAPDH, glyceraldehyde 3-phosphate dehydrogenase; APC, activated protein C; IL, interleukin; LPS, lipopolysaccharide; RT-PCR, reverse transcription polymerase chain reaction; n, number.
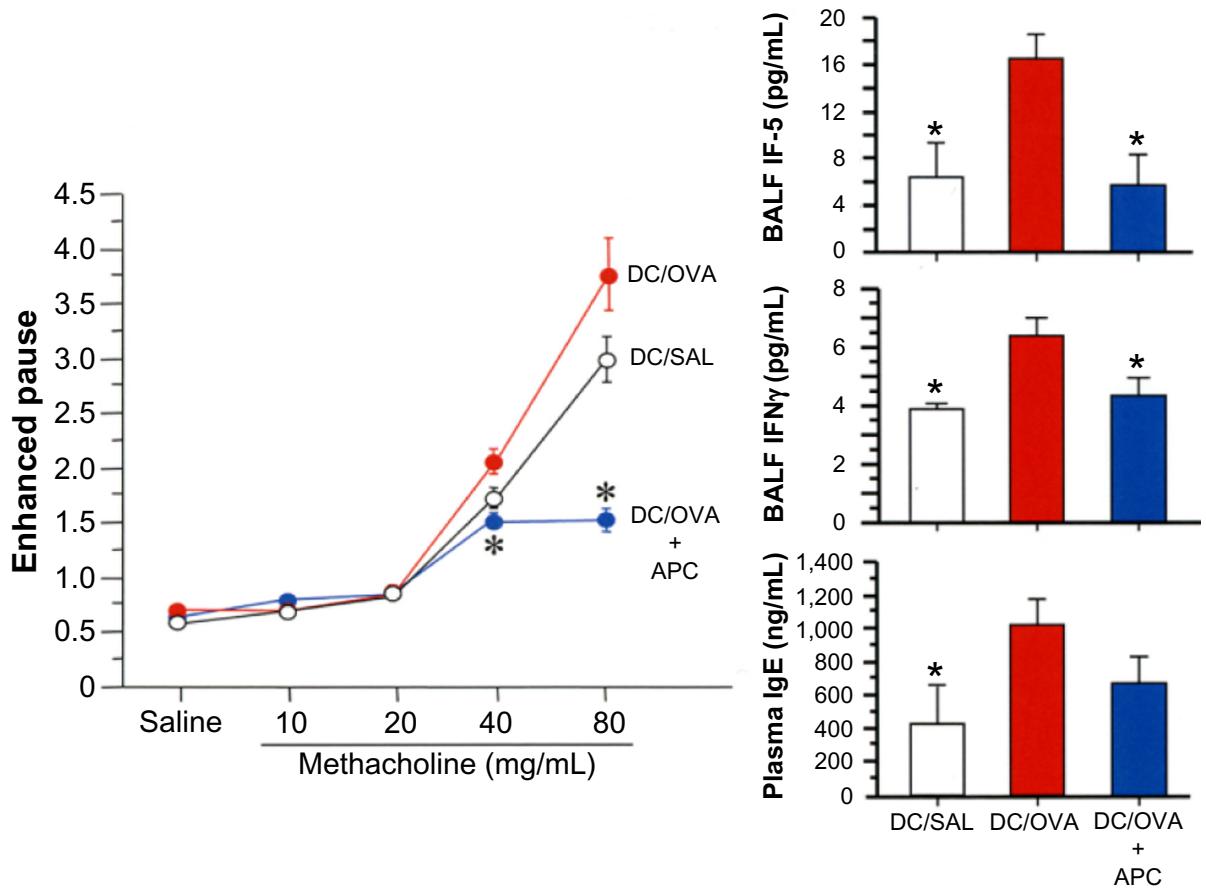

Figure 6 APC inhibits DC activation in vivo.

Notes: Bone marrow-derived DCs were pulsed with OVA, treated with APC, instilled into the trachea of mice, and then the mice were challenged with the antigen. Hyperresponsiveness was measured by enhanced pause and the concentrations of IL-5, IFN $\gamma$, and IgE were measured by ELISA. Data are expressed as the mean \pm standard error of the mean $(n=3)$. The figure shows the representative result from one of two independent experiments. $* P<0.05$ compared to the DC/OVA group.

Abbreviations: DC, dendritic cell; OVA, ovalbumin; SAL, saline; APC, activated protein C; BALF, bronchoalveolar lavage fluid; IL, interleukin; IFN, interferon; Ig, immunoglobulin; ELISA, enzyme-linked immunosorbent assay; n, number. 


\section{Differentiation/maturation of DC subsets and APC}

In the present study, the stimulation of murine bone marrow progenitor cells with pharmacological concentrations of APC at early stages of differentiation was associated with an enhanced number of plasmacytoid DCs and the increased expression of type I IFNs (IFN $\alpha$ and IFN $\beta$ ). The plasmacytoid DC subset is tolerogenic by inducing anergy or the expansion of regulatory T-cells and plays a critical role against viral infections by characteristically secreting large amounts of type I interferons, ${ }^{1,2}$ thus, increased differentiation of plasmacytoid DCs by APC may be important for the regulation of an excessive immune response or for enhancing the control of a viral infection. On the other hand, the conventional DC subset matures after contact with, and phagocytosis of, foreign antigens before migrating to lymphoid tissues where it interacts with T-cells. ${ }^{2}$ In the present study, when murine bone marrow-derived DCs were treated with APC before maturation in the presence of LPS, conventional DCs remained immature with a low expression of cell surface maturation markers and a reduced ability to secrete inflammatory cytokines, indicating that APC can also modulate the immune response by decreasing the maturation of conventional DCs. The maturation markers and the secretion of TNF $\alpha$ were also reduced in human monocyte-derived DCs in the presence of APC, suggesting the relevance of the present findings in human conditions as well.

\section{Differentiation of T-cells and APC}

After maturation, DCs migrate to lymphoid tissues where they interact and activate T-cells. ${ }^{2}$ To evaluate the role of APC during the immunological synapses between DCs and T-cells at lymphoid tissues, in the present study, murine bone marrow-derived DCs were cocultured with CD4 T-cells in the presence of APC under Th1 or Th2 conditions, and the results showed that pharmacological concentrations of APC significantly decreased the number of IFN $\gamma^{+}, \mathrm{IL}_{-} 4^{+}$, and IL- $10^{+} \mathrm{T}$-cells, suggesting the modulatory activity of APC on the immunological synapses. This observation is consistent with findings reported in previous studies showing that APC can modulate the acquired immune response by decreasing the migration of lymphocytes or eosinophils by reducing the expression of cytokines from T-cells, or by increasing the expansion of regulatory T-cells. ${ }^{7,12,13}$

The effect of APC on several cells including DCs is receptor-mediated, as previously described. ${ }^{5,14,15}$ In the present study, the APC receptors EPCR, PAR-1, and CD11b were detected on DCs and are consistent with findings from previous studies. ${ }^{6,16-19}$ To evaluate whether the effect of APC on the immunological synapses is receptor-mediated, OVA-pulsed DCs were pretreated with the antibody to each receptor and cocultured with T-cells in the presence of APC. The results showed that the antibody to each receptor significantly increased the generation of IFN $\gamma^{+} \mathrm{CD} 4$ T-cells, as well as the expression of Th1 and Th2 cytokines when compared to the APC group, indicating the receptormediated action of APC. The fact that mice receiving adoptive transfer of OVA-pulsed DCs pretreated with APC had significantly decreased airway hyperresponsiveness, significantly less lung concentrations of Th1 and Th2 cytokines, and a lower plasma concentration of $\operatorname{IgE}$ after the antigen challenge when compared to the control animals suggests the relevance of this modulatory activity of APC on DCs in in vivo conditions.

\section{Conclusion}

In brief, this study shows that pharmacological doses of APC can modulate the differentiation and maturation of DCs and the polarization of T-cells through its different receptors, suggesting its potential application for the treatment of allergic diseases.

\section{Acknowledgment}

This study was supported by a grant-in-aid (number 26860605) from the Ministry of Education, Culture, Sports, Science, and Technology of Japan. The funders had no role in the study design, data analysis, decision to publish, or preparation of the manuscript.

\section{Disclosure}

Etsuko Harada is an employee of the Iwade Research Institute of Mycology, and Takahiro Matsumoto is an employee of BONAC Corporation. The other authors report no conflicts of interest in this work.

\section{References}

1. Boltjes A, van Wijk F. Human dendritic cell functional specialization in steady-state and inflammation. Front Immunol. 2014;5:131.

2. Kadowaki N. The divergence and interplay between $\mathrm{pDC}$ and $\mathrm{mDC}$ in humans. Front Biosci (Landmark Ed). 2009;14:808-817.

3. Randolph GJ, Ochando J, Partida-Sánchez S. Migration of dendritic cell subsets and their precursors. Annu Rev Immunol. 2008;26:293-316.

4. Suzuki K, Gabazza EC, Hayashi T, Kamada H, Adachi Y, Taguchi O. Protective role of activated protein $\mathrm{C}$ in lung and airway remodeling. Crit Care Med. 2004;32(5 Suppl):S262-S265.

5. Castellino FJ, Ploplis VA. The protein C pathway and pathologic processes. J Thromb Haemost. 2009;7 Suppl 1:140-145.

6. Rezaie AR. The occupancy of endothelial protein $\mathrm{C}$ receptor by its ligand modulates the par-1 dependent signaling specificity of coagulation proteases. IUBMB Life. 2011;63(6):390-396. 
7. Yuda H, Adachi Y, Taguchi O, et al. Activated protein $\mathrm{C}$ inhibits bronchial hyperresponsiveness and Th2 cytokine expression in mice. Blood. 2004;103(6):2196-2204.

8. Xu Y, Zhan Y, Lew AM, Naik SH, Kershaw MH. Differential development of murine dendritic cells by GM-CSF versus Flt3 ligand has implications for inflammation and trafficking. J Immunol. 2007;179(11): 7577-7584.

9. Chapuis F, Rosenzwajg M, Yagello M, Ekman M, Biberfeld P, Gluckman JC. Differentiation of human dendritic cells from monocytes in vitro. Eur J Immunol. 1997;27(2):431-441.

10. Angelov GS, Tomkowiak M, Marçais A, Leverrier Y, Marvel J. Flt3 ligand-generated murine plasmacytoid and conventional dendritic cells differ in their capacity to prime naive CD8 T cells and to generate memory cells in vivo. J Immunol. 2005;175(1):189-195.

11. Yasui H, Gabazza EC, Tamaki S, et al. Intratracheal administration of activated protein $\mathrm{C}$ inhibits bleomycin-induced lung fibrosis in the mouse. Am J Respir Crit Care Med. 2001;163(7):1660-1668.

12. Feistritzer C, Mosheimer BA, Sturn DH, Riewald M, Patsch JR, Wiedermann CJ. Endothelial protein $\mathrm{C}$ receptor-dependent inhibition of migration of human lymphocytes by protein $\mathrm{C}$ involves epidermal growth factor receptor. J Immunol. 2006;176(2):1019-1025.
13. Xue M, Dervish S, Harrison LC, Fulcher G, Jackson CJ. Activated protein $\mathrm{C}$ inhibits pancreatic islet inflammation, stimulates $\mathrm{T}$ regulatory cells, and prevents diabetes in non-obese diabetic (NOD) mice. J Biol Chem. 2012;287(20):16356-16364.

14. Rezaie AR. Regulation of the protein $C$ anticoagulant and antiinflammatory pathways. Curr Med Chem. 2010;17(19):2059-2069.

15. Shimizu S, Gabazza EC, Taguchi O, et al. Activated protein $\mathrm{C}$ inhibits the expression of platelet-derived growth factor in the lung. Am J Respir Crit Care Med. 2003;167(10):1416-1426.

16. Cao C, Gao Y, Li Y, Antalis TM, Castellino FJ, Zhang L. The efficacy of activated protein $\mathrm{C}$ in murine endotoxemia is dependent on integrin CD11b. J Clin Invest. 2010;120(6):1971-1980.

17. Esmon CT. Protein C anticoagulant system - anti-inflammatory effects. Semin Immunopathol. 2012;34(1):127-132.

18. Kerschen E, Hernandez I, Zogg M, et al. Activated protein C targets $\mathrm{CD} 8+$ dendritic cells to reduce the mortality of endotoxemia in mice. J Clin Invest. 2010;120(9):3167-3178.

19. Toltl LJ, Beaudin S, Liaw PC; Canadian Critical Care Translational Biology Group. Activated protein C up-regulates IL-10 and inhibits tissue factor in blood monocytes. J Immunol. 2008;181(3):2165-2173.

\section{Publish your work in this journal}

The Journal of Asthma and Allergy is an international, peer-reviewed open-access journal publishing original research, reports, editorials and commentaries on the following topics: Asthma; Pulmonary physiology; Asthma related clinical health; Clinical immunology and the immunological basis of disease; Pharmacological interventions and

\section{Dovepress}

new therapies. Issues of patient safety and quality of care will also be considered. The manuscript management system is completely online and includes a very quick and fair peer-review system, which is all easy to use. Visit http://www.dovepress.com/testimonials.php to read real quotes from published authors. 